\title{
Variational analysis of self-focusing of intense ultrashort pulses in gases
}

\author{
E. Arévalo and A. Becker \\ Max-Planck-Institut für Physik komplexer Systeme \\ Nöthnitzer Strasse 38, D-01187 Dresden, Germany
}

November 6, 2018

\begin{abstract}
By using perturbation theory we derive an expression for the electrical field of a Gaussian laser pulse propagating in a gas medium. This expression is used as a trial solution in a variational method to get quasianalytical solutions for the width, intensity and self-focusing distance of ultrashort pulse. The approximation gives an improved agreement with results of numerical simulations for a broad range of values of the input power of the pulse than previous analytical results available in the literature.
\end{abstract}

\section{Introduction}

The propagation of high-peak power femtosecond laser pulses in optical media has attracted considerable attention recently [1, 2]. These pulses undergo dramatic changes in their temporal, spatial and spectral properties due to their nonlinear interaction with the medium. The most fundamental process is self-focusing which causes the pulse to be compressed in space, resulting in an increase of the peak intensity [3. Self-focusing would result in a catastrophic collapse of the pulse, but the self-focusing process is usually balanced by multiphoton ionization or excitation of the atoms or molecules in the medium, since the resulting (quasi-)free electron density defocuses the pulse.

The analysis of optical pulse propagation is usually based on the description of the pulse in terms of its complex field envelope, neglecting the underlying rapid oscillations at its carrier frequency. The resulting slowly 
varying envelope approximation reduces the Maxwell's equations to higher dimensional nonlinear Schödinger equations (NLSE). These equations are not integrable, so they do not have soliton solutions. However, they possess stationary solutions which are unstable on propagation. The self-focusing distance is determined as the (first) point of infinite intensity in the solution of the NLSE.

Most of the quantitative analysis of self-focusing (and further propagation) of the pulse results from numerical computation. For example, from a curve-fitting procedure based on numerical simulations a popular analytical formula for the location of the first singularity (self-focusing distance) for the propagation of a CW laser beam has been given by Dawes and Marburger [4, 3]:

$$
\xi_{s f}^{(M)}=\frac{0.367}{\sqrt{(\sqrt{\mathcal{P}}-0.852)^{2}-0.0219}},
$$

where $\mathcal{P}$ is the input power scaled in units of the critical power for selffocusing, $P_{\text {crit }}=\lambda_{0}^{2} / 2 \pi n_{0} n_{2}$, with $\lambda_{0}$ is the wavelength and $n_{0}$ and $n_{2}$ are the linear and nonlinear indices of refraction, respectively [5]. Formula (11) has been verified in many experiments and numerical simulations. Besides its accurateness and usefulness for estimations it however does not give much insight in the physics of the underlying process.

In order to get a deeper insight into a physical process, it is often useful to use approximative theories, even if full numerical solutions are available. A few approximations have been discussed already in the early review paper on self-focusing by Marburger [3]. The resulting expressions provide, however, rather qualitative than quantitative estimations only. Alternative approaches have been proposed to analyze the effect of self-focusing, namely a systematic perturbation theory [6, 7], a ray-equation approximation [3], an approach based on Fermat's principle [8], a variational analysis [9], a paraxial parabolic approximation [10], or a source-dependent expansion method [1]. The predictions of these models for the self-focusing distance agree quantitatively well with the exact solutions from numerical simulations for input powers close to the critical power, but deviate quickly as the power increases.

In this paper we present a quasianalytical approximation which provides a description of the self-focusing phenomenon not only qualitatively but also quantitatively. It is based on the variational approach using a trial solution, which contains a first-order perturbation correction of the phase resulting from the transverse distortion of the pulse. As we show below, predictions for the self-focusing distance, the on-axis peak intensity and the radius of the pulse within this ansatz are in better agreement with those of numerical simulations over a broad range of input powers than previous analytical 
results available in the literature. We show how the pulse separates into an inner and an outer self-trapped component along the propagation distance. The inner component self-focus, while the outer one stays as background.

The paper is organized as follows: First, we derive the phase correction of the pulse using first-order perturbation theory. The corrected form of the pulse is then used as trial solution in the Lagrangian of the system to obtain equations for the self-focusing distance, the on-axis peak intensity and the radius of the pulse. Next, the predictions of the approximation will be compared with those of the earlier models and of numerical simulations. Finally, an analogy with the problem of a particle in a finite quantum well is presented. In this analogy, the bound state and its exponential-decaying wings penetrating a smooth potential wall correspond to the inner and outer component of the pulse, respectively. The self-focusing is associated with the shrinking of the width of the quantum well.

\section{Variational approach}

We study the propagation of a linearly polarized laser beam in gases. For the explicit calculations we do not restrict to any specific gas. Our analysis is based on the scalar wave equation, which can be obtained from the Maxwell equations (e.g. [8]):

$$
\partial_{z}^{2} E+\Delta E-\frac{\epsilon_{0}}{c^{2}} \partial_{t}^{2} E=\frac{4 \pi \chi^{(3)}}{c^{2}} \partial_{t}^{2} E^{3}+\frac{4 \pi}{c^{2}} \partial_{t} J
$$

Here, $\chi^{(3)}$ is the third order nonlinear susceptibility coefficient of the medium, $\epsilon_{0}$ is the linear dielectric constant of the gas and $\mathrm{c}$ is the speed of light. Since we are interested in the dynamics of the pulse up to the self-focusing distance only, we neglect the plasma effects $(J=0)$, which usually balance the catastrophic collapse and, hence, influence mainly the propagation of the pulse after the self-focusing point. Using the slowly varying envelope approximation, Eq. (2) can be written in the retarded coordinate frame $\left(t \rightarrow t-z / v_{g}\right)$ as:

$$
\begin{aligned}
& i \partial_{\xi} u+\frac{1}{4} \Delta_{\perp} u+\frac{L_{D}}{L_{n l}}|u|^{2} u \\
& -\frac{1}{4} \frac{L_{D}}{L_{d}}\left(\partial_{\tau}^{2} u+\frac{i}{3} \frac{L_{d}}{L_{d}^{\prime}} \partial_{\tau}^{3} u\right)=0
\end{aligned}
$$

where $u=\mathcal{E} / \sqrt{I_{0}}, \xi=z / L_{D}, \tau=t / T_{0}$, and the transverse coordinate $r$ is given in units of the length of the pulse $w_{0}\left(w_{0}\right.$ is the radius at $1 / e^{2}$ of 


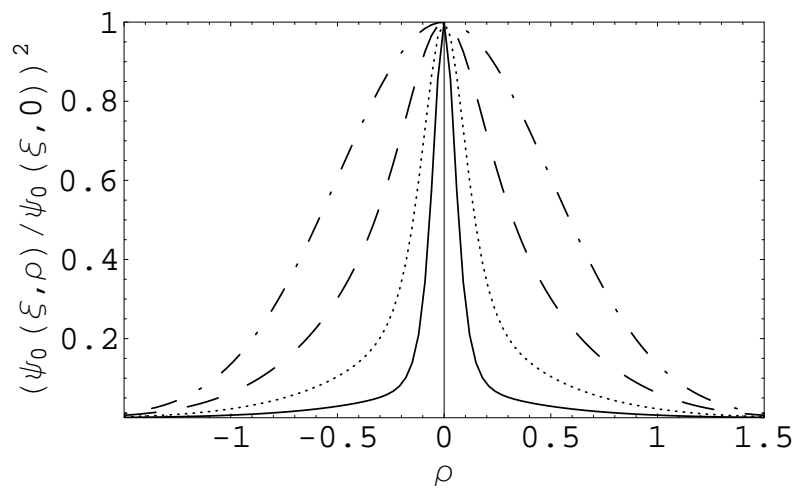

Figure 1: Results of numerical simulations for the normalized pulse shape for $P / P_{c r}=5: \xi=0$ (dotted-dashed line), $\xi=0.15$ (dashed line), $\xi=0.20$ (dotted line), $\xi=0.23$ (solid line).

the irradiance). The characteristic lengths are given by $L_{D}=k_{z} w_{0}^{2} / 2, L_{d}=$ $T_{0}^{2} / 2 k_{2}, L_{d^{\prime}}=T_{0}^{3} / 2 k_{3}$ and $L_{n l}=\left(n_{2} k_{0} I_{0}\right)^{-1}$, where $k_{0}=2 \pi / \lambda, k_{z}=n_{0} k_{0} . k_{2}$ and $k_{3}$ are the second and third order group-velocity dispersion coefficients, respectively, $T_{0}$ is the duration of the pulse $\left(T_{F W H M}=\sqrt{2 \log 2} T_{0}\right)$, and $I_{0}$ is the input peak intensity (in units of $\mathrm{W} / \mathrm{cm}^{2}$ ). We further neglect in Eq. (3) the effect of the group velocity dispersion to get the well-known $(2+1)$-dimensional NLSE,

$$
i \partial_{\xi} u+\frac{1}{4} \Delta_{\perp} u+F\left[u u^{*}\right] u=0,
$$

where the star stands for complex conjugation and $F\left[u u^{*}\right]=c_{1}|u|^{2}$ with $c_{1}=L_{D} / L_{n l}$. During the early stage of propagation of the pulse (up to the self-focusing point) in gases the effect of the group velocity is negligible, namely if $L_{n l} \ll L_{d}<L_{d^{\prime}}$. Thus, for the present analysis we expect that the condition is fulfilled as long as the self-focusing distances are small, i.e. as larger the input power of the beam is.

\subsection{Phase correction}

The variational method has been applied to the $(2+1)$-dimensional NLSE recently [9] to study qualitatively the phenomenon of self-focusing in air. As mentioned at the outset the method is as good as the trial solution. Usually the self-similarity assumption is used in order to integrate over the transversal coordinates taking into account that the total power of the NLSE is invariant. This is done by assuming that the shape of the pulse remains unchanged up to the self-focusing point. The assumption preserves the lens transformation 
of the NLSE. However, from numerical simulations one can observe that the initial pulse shape gets distorted during the self-focusing process (c.f. Fig. 11). In fact, the pulse separates into two components as it propagates [7],

$$
u(\xi, r)=\psi_{0}(\xi, r)+\epsilon \psi_{1}(\xi, r),
$$

where $\psi_{0}$ is the high intensity inner core of the pulse, which self-focuses and $\epsilon \psi_{1}$ is the low intensity outer part, which propagates forward following the linear propagation mode. In recent experiments and numerical simulations [12, 13, 14, 15] it has been shown that this weak large background plays a decisive role in the propagation and filamentation process.

We expect that the self-focusing process is influenced by the interaction between the inner core and the outer part too. To this end we use a trial solution in the variational method, in which the perturbation by the background is taken into account as a correction in the phase. We first separate amplitude and phase of the pulse as:

$$
\begin{aligned}
u(\xi, r)= & |u(\xi, r)| \exp (i S(\xi, r)) \\
= & \left|\psi_{0}(\xi, r)\right| \exp \left(i S_{0}(\xi, r)\right) \\
& +\epsilon\left|\psi_{1}(\xi, r)\right| \exp \left(i S_{1}(\xi, r)\right),
\end{aligned}
$$

and by substituting in Eq. (41) we get up to order $\epsilon$ that

$$
S(\xi, r)=\sum_{j=0}^{1} S_{j}(\xi, r)+O\left(\epsilon^{2}\right)
$$

with

$$
S_{j}(\xi, r)=S_{j}(0, r)+\int_{0}^{\xi} n_{j}(\xi, r) d \xi, \quad j=0,1 .
$$

Here,

$$
\begin{aligned}
n_{0}(\xi, r)= & F\left[\left|\psi_{0}(\xi, r)\right|^{2}\right]-\frac{1}{4}\left(\partial_{\xi} S(\xi, r)\right)^{2} \\
& +\frac{\Delta_{\perp}\left|\psi_{0}(\xi, r)\right|}{\left|\psi_{0}(\xi, r)\right|}
\end{aligned}
$$


and

$$
\begin{aligned}
n_{1}(\xi, r) & =\epsilon\left\{\left(2 F^{\prime}\left[\left|\psi_{0}(\xi, r)\right|^{2}\right]\left|\psi_{0}(\xi, r)\right|\left|\psi_{1}(\xi, r)\right|\right.\right. \\
- & \frac{\left|\psi_{1}(\xi, r)\right| \Delta_{\perp}\left|\psi_{0}(\xi, r)\right|}{4\left|\psi_{0}(\xi, r)\right|^{2}}+\frac{\Delta_{\perp}\left|\psi_{1}(\xi, r)\right|}{4\left|\psi_{0}(\xi, r)\right|} \\
- & \left.\frac{\left|\psi_{1}(\xi, r)\right|}{4\left|\psi_{0}(\xi, r)\right|}\left(\partial_{r} S(\xi, r)\right)^{2}\right) \\
\times \quad & \cos \left(S_{0}(\xi, r)-S_{1}(\xi, r)\right) \\
+ & \quad \frac{\left|\psi_{1}(\xi, r)\right| \Delta_{\perp} S_{1}(\xi, r)}{4\left|\psi_{0}(\xi, r)\right|} \\
+ & \left.\frac{\partial_{r} \psi_{1}(\xi, r) \partial_{r} S_{1}(\xi, r)}{2\left|\psi_{0}(\xi, r)\right|}\right) \\
\times & \left.\sin \left(S_{0}(\xi, r)-S_{1}(\xi, r)\right)\right\} .
\end{aligned}
$$

The desired trial solution is then given by

$$
u(\xi, r)=\psi_{0}(\xi, r) \exp \left(i S_{1}(\xi, r)\right)
$$

with $S_{1}(0, r)=0$. Here the amplitude corrections of order $\epsilon$ have been neglected, while phase corrections have been kept. Note that $u(\xi, r)$ still fulfils the self-similarity assumption, namely $|u(\xi, r)|^{2}=\left|\psi_{0}(\xi, r)\right|^{2}$.

The phase correction $S_{1}(\xi, r)$ is obtained using first-order perturbation theory as follows. Inserting Eq. (5) into Eq. (4) and collecting powers of $\epsilon$ we get

$$
\begin{aligned}
\epsilon^{0} \quad: \quad & i \partial_{\xi} \psi_{0}+\frac{1}{4} \Delta_{\perp} \psi_{0}+F\left[\psi_{0} \psi_{0}^{*}\right] \psi_{0}=0, \\
\epsilon^{1}: & i \partial_{\xi} \psi_{1}+\frac{1}{4} \Delta_{\perp} \psi_{1}+F\left[\psi_{0} \psi_{0}^{*}\right] \psi_{1} \\
& +F^{\prime}\left[\psi_{0} \psi_{0}^{*}\right] \psi_{0} \psi_{0}^{*} \psi_{1}+F^{\prime}\left[\psi_{0} \psi_{0}^{*}\right] \psi_{0}^{2} \psi_{1}=0,
\end{aligned}
$$

where the prime stands for the derivative with respect to the argument. Note, that the solution of Eq. (13) has the form

$$
\psi_{1}=\partial_{a(\xi)} \psi_{0},
$$

where $a(\xi)$ is the length of the pulse. Thus, the phase correction can be obtained for any shape of the inner core by taking its derivative and separating the phase. 
We do not consider the presence of any external perturbation, like losses or ionization. However, the theory presented here can be extended considering these terms following the same lines as in [9]. We have checked carefully that the predictions of the numerical calculations for the self-focusing distance do vary by less than $1 \%$, if we include external perturbations. Other properties, such as the pulse length or the on-axis intensity (up to the self-focusing point) are even less sensitive to these effects. It is therefore justified, for the sake of simplicity, to neglect external perturbations.

\subsection{The Gaussian pulse shape}

As outlined above, the phase correction can be obtained for any shape of the pulse. In order to investigate the effect we apply the theory to the most important case of a Gaussian pulse shape below. In this case

$$
\psi_{0}(\xi, r)=\frac{A}{a(\xi)} \exp \left(\rho^{2}\right) \exp \left(i b_{0} a(\xi) \rho^{2}\right),
$$

where $\rho=r / a(\xi)$ and $b_{0}$ is a constant describing the initial wave front divergence of the pulse (i.e. $b_{0}=0$ for collimated pulse, or $b_{0}=-1 / f$ for the case of an external lens with focal length $f$ ), we obtain the phase correction from the solution of Eq. (13),

$$
\begin{aligned}
\psi_{1}(\xi, r)= & \frac{A}{a^{2}(\xi)} \exp \left(-\rho^{2}\right) \exp \left(i b_{0} a(\xi) \rho^{2}\right) \\
& \times\left(2 \rho^{2}-i b_{0}, a(\xi) \rho^{2}-1\right),
\end{aligned}
$$

as follows. Substituting Eqs. (15) and (16) into (91) and (10) we get

$$
\begin{aligned}
n_{0}(\xi, r) & =-\frac{1}{4}\left(\partial_{r} S(\xi, r)\right)^{2} \\
& +\quad \frac{1}{a^{2}(\xi)}\left(\rho^{2}-1+A^{2} c_{1} \exp \left(-2 \rho^{2}\right)\right)
\end{aligned}
$$

and

$$
\begin{aligned}
n_{1}(\xi, r) & =\frac{2 A^{2} c_{1} \epsilon}{a^{3}(\xi)}\left(2 \rho^{2} \exp \left(-2 \rho^{2}\right)-\exp \left(-2 \rho^{2}\right)\right) \\
+ & \frac{2 \epsilon}{a^{3}(\xi)}\left(1-2 \rho^{2}\right)
\end{aligned}
$$


where $a(\xi)$ is an undetermined function. In Ref. [6] it has been shown that $a(\xi)$ can be determined using

$$
\beta=-a^{3}(\xi) a^{\prime \prime}(\xi)
$$

which is proportional to the excess power, as long as the excess power is small. With this relation one is able to solve the integral (8) in terms of $\beta$ for Gaussian pulses. However, the solution of the integral is ill-posed, since the integrals in the solution diverge. Therefore, we have adopted an adiabatic approximation instead, namely that the transverse form of $n_{1}(\xi, r)$ remains unaffected along the propagation distance, i.e

$$
\begin{aligned}
S_{1}(\xi, r) & =n_{1}(\xi, r) \xi \\
& =\frac{2 A^{2} c_{1} \epsilon \xi}{a^{3}(\xi)}\left(2 \rho^{2} \exp \left(-2 \rho^{2}\right)-\exp \left(-2 \rho^{2}\right)\right) \\
& +\frac{2 \epsilon \xi}{a^{3}(\xi)}\left(1-2 \rho^{2}\right) .
\end{aligned}
$$

The last term of Eq. (21) arises due to the diffraction term in Eq. (13) for the outer part of the pulse. This term should not effect the propagation of the inner core part and we neglect it. The validity of this assumption can be shown using the variational method (see Appendix A). Thus, finally we get

$$
\begin{aligned}
& u(\xi, r)=\frac{1}{a(\xi)} A \exp \left(\rho^{2}\right) \\
& \times \quad \exp \left(i b_{0} a(\xi) \rho^{2}\right) \exp \left(i S_{1}(\xi, r)\right)
\end{aligned}
$$

with

$$
S_{1}(\xi, r)=\frac{2 A^{2} c_{1} \epsilon \xi}{a^{3}(\xi)}\left(2 \rho^{2}-1\right) \exp \left(-2 \rho^{2}\right)
$$

\subsection{Lagrangian and variational analysis}

The Lagrangian functional for the system (4) reads,

$$
\mathcal{L}=\frac{i}{2}\left(u \partial_{\xi} u^{*}-u^{*} \partial_{\xi} u\right)+\frac{1}{4}\left|\partial_{\xi} u\right|^{2}-\frac{1}{2} c_{1}|u|^{2} .
$$

From Eqs. (22) and (46) we define

$$
\begin{aligned}
u(\xi, r)= & \frac{1}{a(\xi)} A(\xi) \exp \left(-\rho^{2}\right) \exp \left(i b_{0} a(\xi) \rho^{2}\right) \\
& \times \exp (i \phi(\xi)) \exp \left(i S_{1}(\xi, r)\right)
\end{aligned}
$$


with

$$
S_{1}(\xi, r)=c(\xi) a(\xi)\left(2 \rho^{2}-1\right) \exp \left(-2 \rho^{2}\right),
$$

where the variational parameter $A(\xi), a(\xi), c(\xi)$ and $\phi(\xi)$ are sufficient to describe the dynamics of the problem. Here, we consider a collimated input laser pulse, focused by a lens, given by

$$
u(0, r)=u_{0} \exp \left(-\tau^{2}\right) \exp \left(-r^{2}\right) \exp \left(-i \frac{r^{2}}{f}\right) .
$$

where $f$ is the focal length of the lens in units of diffraction length $L_{D}$. Note that the temporal dependence of the variational parameters in (25) is not written explicitly. Inserting Eq. (25) into the Lagrangian (24) and integrating over the transverse coordinate we obtain the reduced Lagrangian

$$
\langle\mathcal{L}\rangle=2 \pi \int_{0}^{\infty} \mathcal{L} r d r
$$

which depends on the variational parameters and the independent variables $\xi$ and $\tau$ only. The equations of motion for the variational parameters are given by

$$
\frac{d}{d \xi} \partial_{\left(\partial_{\xi} \mu_{i}\right)}\langle\mathcal{L}\rangle-\partial_{\mu_{i}}\langle\mathcal{L}\rangle=0,
$$

where $\mu_{i}=A, a, c, \phi(\mathrm{i}=1, \ldots, 4)$. This leads to the following set of coupled equations:

$$
\begin{gathered}
a^{\prime}(\xi)=\frac{16}{9} c(\xi)+b_{0}, \\
c^{\prime}(\xi)=-\frac{2(\mathcal{P}(\xi)-1)}{a^{3}(\xi)}, \\
\mathcal{P}^{\prime}(\xi)=0,
\end{gathered}
$$

where $\mathcal{P}(\xi)=P(\xi) / P_{c r}$ with $P(\xi)=2 \pi \int_{0}^{\infty}|u| r d r=\pi A^{2}(\xi) / 2$, and the critical power $P_{c r}=\pi / c_{1}$.

From Eqs. (30)- - with the initial conditions, $a(0)=1$ and $c(0)=0$, we get

$$
a^{3}(\xi) a^{\prime \prime}(\xi)=-\beta=-\frac{32}{9}(\mathcal{P}-1) .
$$


or

$$
a(\xi)=\sqrt{\left(1-b_{0} \xi\right)^{2}-\beta \xi^{2}}
$$

The self-focusing distance,

$$
\xi_{s f}=\frac{1}{\sqrt{\beta}-b_{0}}
$$

is obtained for the collapse of the pulse $(a(\xi)=0)$. Note that

$$
\frac{1}{\xi_{s f}}=\frac{1}{\xi_{s f}\left(b_{0}=0\right)}+\frac{1}{f}
$$

in agreement with the lens transformation property of the NLSE [3, 7]. The on-axis intensity is given by

$$
\begin{aligned}
I(\xi) & =|u(\xi, 0)|^{2}=\frac{A^{2}(\xi)}{a^{2}(\xi)} \\
& =\frac{A^{2}(\xi)}{\left(1-b_{0} \xi\right)^{2}-\beta \xi^{2}} .
\end{aligned}
$$

\section{Results and comparisons}

Below we present the predictions of the present theoretical ansatz and compare them with those of other semi-analytical estimations and the results of numerical calculations using Eq. (41). The comparisons are performed for the case of a collimated Gaussian pulse without external focusing. We should note that this is the most extreme case. The presence of a finite external focusing reduces the relative errors between the theory and simulations.

First, we concentrate on the results of the present ansatz for the selffocusing distance, $\xi_{s f}$ (c.f. Eq. (36) ). In Fig. 2 the relative error (solid line),

$$
\eta_{s f}=\frac{\xi_{s f}-\xi_{s f}^{(M)}}{\xi_{s f}^{(M)}}
$$

with respect to Marburger's formula, Eq. (11), is shown as a function of the input power (scaled in units of the critical power). Note that Eq. (11) has been derived from numerical simulations and is, hence, an excellent estimate of the exact self-focusing distance. The comparison shows that the relative error is largest (about 40\%) for input powers close to the critical power and 


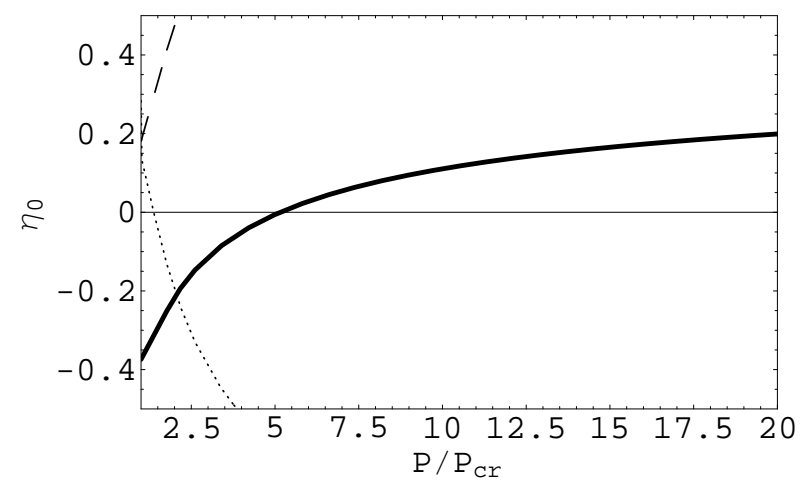

Figure 2: Relative error $\eta_{s f}$ of the predictions of the present theory for the self-focusing distance (solid line) with respect to the Marburger's Formula as function of $\mathcal{P}=P / P_{c r}$. Also shown are the relative errors for earlier models (see text).

is less than $20 \%$ for $2.5<\mathcal{P}<20$. The large error near the critical power for self-focusing might be due to the neglect of the group velocity in the analysis.

Also shown in Fig. 2] are the relative errors (with respect to Marburger's formula) for the predictions of the self-focusing distance resulting from analyses by means of either one of the following methods, ray-equation approximation [3], Fermat's principle [8, variational analysis [9], paraxial parabolic approximation [10], or source-dependent expansion method [1] (dashed line):

$$
\xi_{s f}^{(1)}=\frac{1}{\sqrt{\mathcal{P}-1}},
$$

and by using perturbation theory (dotted line, [7]):

$$
\xi_{s f}^{(2)} \sim \frac{2}{\mathcal{P}} \sqrt{\frac{M}{N_{c} \sqrt{\mathcal{P}-1}}},
$$

where the constants $M=0.55$ and $N_{c}=1.86$ are derived from the Townes soliton shape. The value of $\xi_{s f}^{(2)}$ in Eq. (41) is given in diffraction units and therefore four times larger than in [7]. The latter formula, Eq. (41), is valid for $\mathcal{P}<2$.

Both estimations are in excellent agreement with the results of Marburger's formula near the critical power but strongly deviate as the power increases at larger powers. It is clearly seen, that already at $\mathcal{P} \simeq 2$ the predictions within the present theory are in closer quantitative agreement with the exact results. For example, at $\mathcal{P}=10$ the predictions of the earlier theories deviate from the exact result by more than $70 \%$ in the case of perturbation 


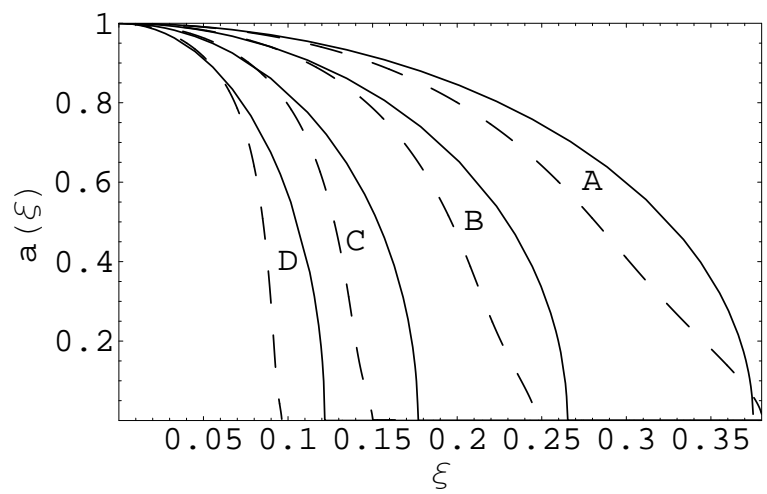

Figure 3: Comparison of the predictions of the present theory (solid lines) for the pulse length with numerical simulations (dashed lines) for $\mathcal{P}=3(\mathrm{~A})$, $\mathcal{P}=5(\mathrm{~B}), \mathcal{P}=10(\mathrm{C}), \mathcal{P}=20(\mathrm{D})$.

theory (see Eq. (41)) or by more than $100 \%$ in the case of other theories (see Eq. (40)), while the present theoretical prediction agree within about $20 \%$ with Marburger's formula. We attribute this to the phase correction obtained from the interaction with the weak large background, which has not been taken into account in the earlier work. In fact, from our ansatz we observe that the background does not diffract and receives energy from the inner part of the pulse during the self-focusing process, as we will discuss below in Section 4. We also mention that the expression in Eq. (40) for the self-focusing can be obtained by taking into account the two first leading terms of the power series of the phase correction in Eq. (26) only. This means that the earlier ansatz (see for instance [9]) is a limiting case of Eqs. (25) and (26).

We find also that the estimations of the present theory and the numerical simulations for the pulse length and the on-axis intensity agree within $30 \%$. In Fig. 3 a comparison of the pulse length $a(\xi)$, Eq. (34) (solid line), and the $1 / e^{2}$-level width of the intensity profile using numerical simulation (dashed line) of a collimated Gaussian pulse for different input powers is shown.

Since the range of values of the ordinate in Fig. 3 is the same (but not those of the abscissa) we have evaluated an relative error using the inverse functions,

$$
\eta_{1}=\frac{\xi\left[a_{\text {theory }}\right]-\xi\left[a_{\text {simulation }}\right]}{\xi\left[a_{\text {simulation }}\right]} .
$$

The results are presented in Fig. 4(a) as a function of $a(\xi)$ for several values of the input power. We observe that $\eta_{1}$ is in general lower than $10 \%$ for $a(\xi) \sim 1$, i.e. in the early stages of the propagation (c.f Fig. 3). Close to 


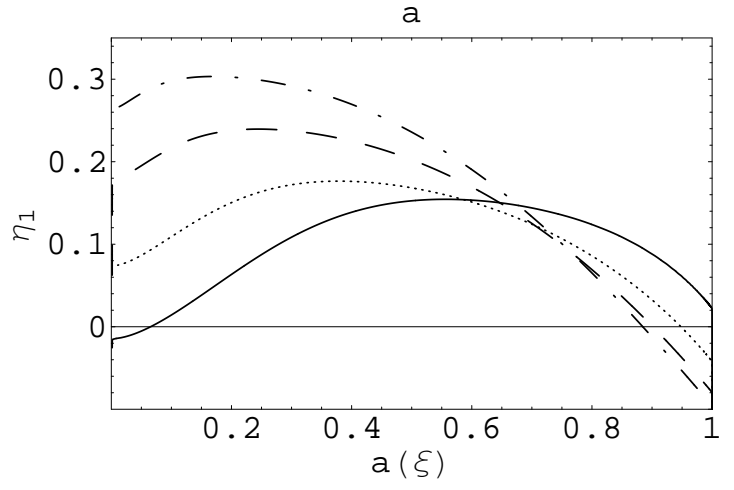

b

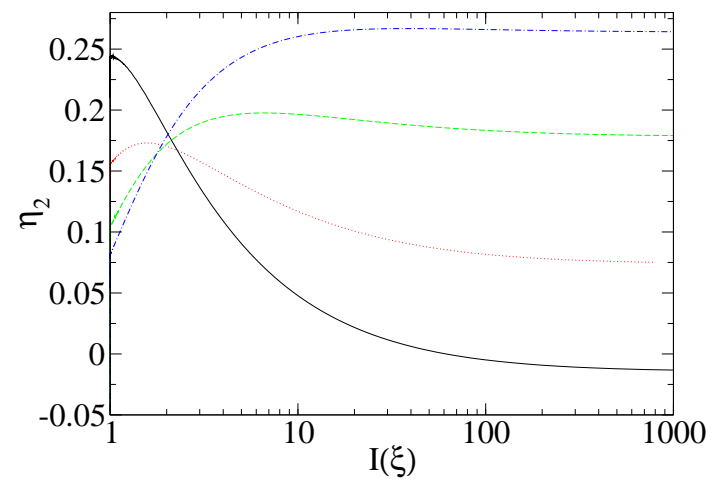

Figure 4: Relative errors $\eta_{1}$ (panel a) and $\eta_{2}$ (panel b) for different input powers, $\mathcal{P}=3$ (solid line), $\mathcal{P}=5$ (dotted line), $\mathcal{P}=10$ (dashed line), $\mathcal{P}=20$ (dotted-dashed line). 


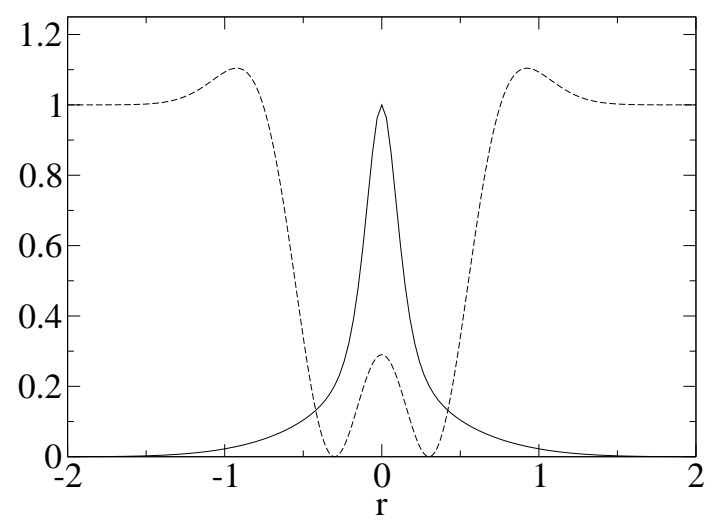

Figure 5: Comparison between the intensity profile of the pulse (solid line) obtained from the simulation and the trapping potential derived from the theory at $\xi=0.2$ with $\mathcal{P}=5$.

the self-focusing point, $a(\xi) \sim 0, \eta_{1}$ increases to above $20 \%$ for high input powers $(\mathcal{P} \geq 10)$. This shows that in the simulations the pulse undergoes a stronger deformation than predicted by the present ansatz.

In the same way we define the relative error for the on-axis intensity, namely

$$
\eta_{2}=\frac{\xi\left[I_{\text {theory }}\right]-\xi\left[I_{\text {simulation }}\right]}{\xi\left[I_{\text {simulation }}\right]}
$$

As can be seen from the results in Fig. 4 (b) $\eta_{2}$ is lower than $20 \%$ for $\mathcal{P} \leq 10$. In general, $\eta_{2}$ tends to be constant for larger values of the intensity.

\section{Quantum well analogy}

In order to further understand the role of the weak large background on the dynamics of the pulse, we have investigated our ansatz using the quantum well analogy. In this picture $\xi$ acts as a fictitious time variable, $u(\xi, r)$ as a bound state and $-\phi^{\prime}(\xi)$ as the energy function of the system. By substituting Eq. (25) into Eq. (41) and taking the real part, one finds an equation for $-\phi^{\prime}(\xi)$. If we neglect the diffraction part of this equation (neglecting kinetic energy in the quantum analogue), it is straight forward to derive a potentiallike function. In Fig. 5 the normalized shape of this potential is compared with the normalized shape of the intensity profile of the pulse obtained from numerical simulations. We observe that the shape of the potential is a well which contains two regions, an inner and an outer part. The inner part extends from the center of the well $(r=0)$ up to the absolute minimum. The outer part includes the rest, namely the potential wall which smoothly grows 


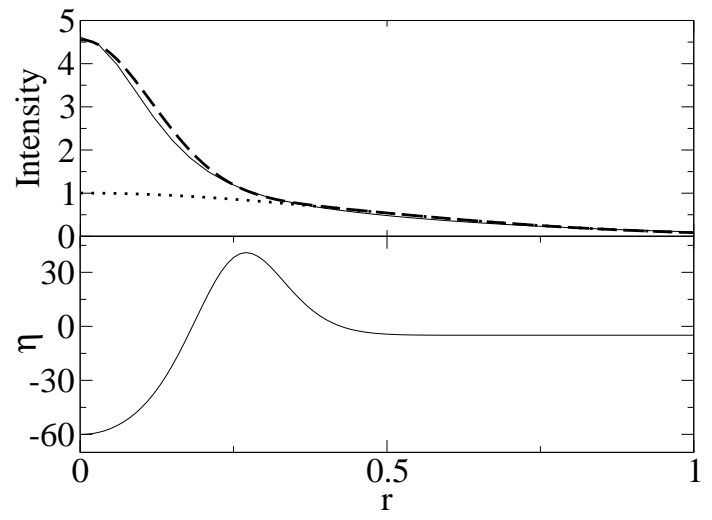

Figure 6: The upper panel contains an example of the intensity distribution obtained from simulation (solid line) for $\mathcal{P}=5$ and $\xi=0.2$. The distribution can be fitted by the superposition of two Gaussians (dashed line). One for the inner part, and the other for the background (dotted line). The lower panel contains the result of the operation in Eq. (44). Note that the region, where the dashed line tends towards the dotted line (inner and outer part join), is located where the maximum of the function $\eta$ is.

radially up to a certain constant value. Notice that the wall is infinitely thick, so no analogue of the tunnel effect is possible here. These features of the potential well reveal already that the pulse possesses two components. In fact, we observe in Fig. 5 that the inner component of the pulse is bell-shaped, while the outer component is an analogue of the exponential-decaying wings of the probability density of a bound state penetrating a smooth potential well. The potential well shrinks radially along the propagation distance and the local maximum in the center decreases and eventually disappears at the self-focusing distance. So, the inner component of the pulse tends to concentrate at the origin and eventually self-focuses.

We have estimated the region in which the inner part of the pulse is concentrated as follows. From the intensity profile of the pulse, obtained from numerical simulations, one can notice that it is well fitted by the superposition of two Gaussian functions, as shown in the upper panel of Fig. [6] In order to determine quantitatively where the inner and outer part join, we have performed the following operation in our simulations:

$$
\eta=\partial_{r}\left(\frac{\partial_{r}|u(\xi, r)|^{2}}{|u(\xi, r)|^{2}}\right),
$$

where $|u(\xi, r)|^{2}$ is the intensity distribution. Note that $\eta$ is constant when 


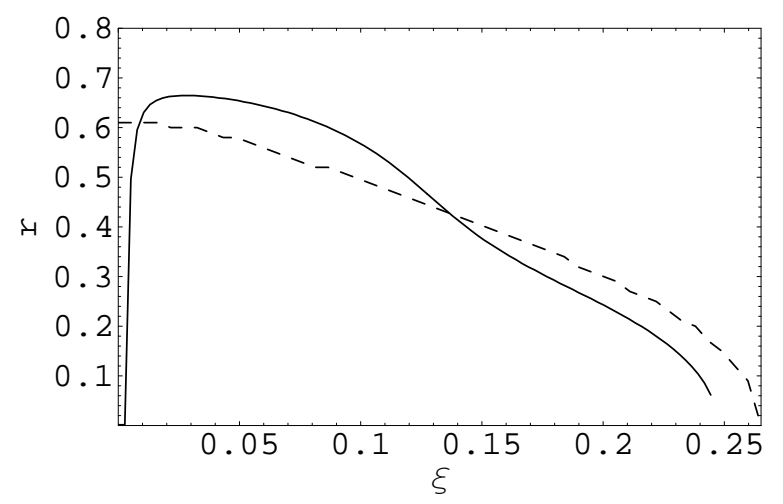

Figure 7: Comparison of the position of the minimum of the potential (dashed line) and the position of the deformation of the Gaussian pulse (solid line) vs. $\xi$ for $\mathcal{P}=5$.

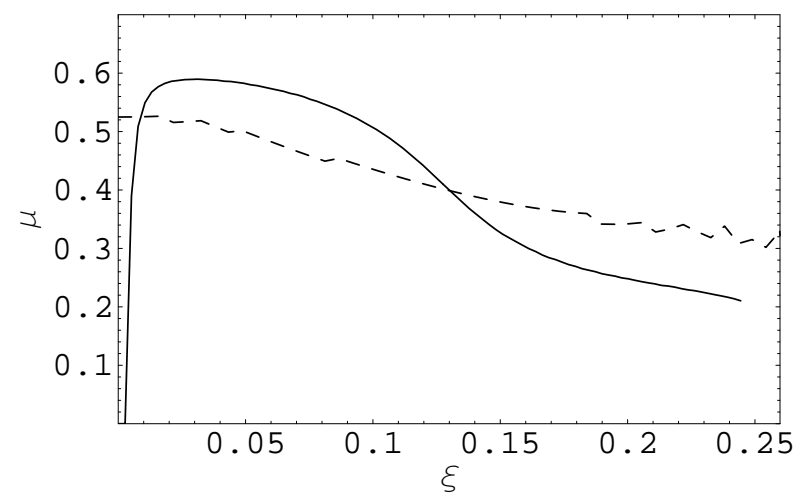

Figure 8: Estimated fraction of energy, $\mu$, that self-focuses for $\mathcal{P}=5$. Solid line: simulation, dashed line: theory.

$u(\xi, r)$ is a pure Gaussian function. For the present case (c.f. upper panel of Fig. 6) $\eta$ has its absolute maximum in the region where the inner and outer part of the pulse join (c.f. lower panel of Fig. 6). Thus, we determine the boundary position, $r_{\text {join }}$, between the inner and outer part of the pulse by the absolute maximum of $\eta$.

In Fig. [7 we show the comparison between $r_{\text {join }}$ from simulations (solid line) and the position of the absolute minimum of the potential obtained from the quantum well potential (dashed line c.f. Fig. 5) Note that at $\xi=0$ no deformation is observed in the simulations since the initial pulse shape is Gaussian. For $\xi>0$ a deformation of the pulse appears at the origin $(r=0)$ and moves quickly to $r \simeq 0.65$. Afterwards $r_{\text {join }}$ shrinks and disappears at the self-focusing distance. This qualitative behavior is found for all the cases 
considered in this paper. Thus, our theoretical model does not predict the appearance of the deformation at the origin, but the position of the absolute minimum of the potential well follows closely the behavior of $r_{\text {join }}$ over the main part of the propagation distance.

From the radii shown in Fig:7] we can calculate the fraction of energy $\mu$ stored in the inner component of the pulse, which is compared in Fig. 8] with those from the numerical simulations. We observe that there is a energy flow from the inner part to the outer part when the pulse shrinks. Note that the inner component of the pulse releases more energy in the second half of its propagation towards the self-focusing distance, where the shrinkage of $r_{\text {join }}$ is more pronounced. At the self-focusing distance most of the energy is stored in the outer component of the pulse which stays with the pulse and does not diffract. We also note that for low powers $(\mathcal{P}<5)$ the present model overestimates the release of energy towards the outer region, while for higher powers $(\mathcal{P}>5)$ it is underestimated. This difference is related to the discrepancy found for the estimation of the self-focusing distance (see Fig. 2). We note, that the ansatz in earlier works (see for instance [9]) would lead to a potential well too, however the wall of this potential well has a finite thickness. Thus, in the previous ansatz the outer part of the pulse diffracts out in contrast to the present model where the pulse is completely self-trapped.

\section{Conclusion}

In conclusion, we have revisited the problem of self-focusing of a laser pulse in a gas Kerr medium modeled by the $(2+1)$-dimensional nonlinear Schrödinger equation. We have defined a new trial solution taking into account that during the self-focusing process the pulse splits in an inner and an outer component. In the trial solution the outer component is taken into account via a phase correction. We have used a quantum well analogy to explain the dynamics of the laser pulse. According to this picture, the laser pulse is represented by a bound state of a particle trapped in a quantum well. The inner component of the laser pulse corresponds to that part of the bound state where the energy of the system is larger than the bottom of the potential well. The outer part is the analogue of the exponential-decaying wings of the bound state penetrating a smooth potential wall. Here, the self-focusing process of the laser pulse is associated to the shrinkage of the width of the quantum well. Theory and simulations show that during the self-focusing process the energy of the inner component flows to the outer one which stays with the pulse and does not diffract. Finally, only a fraction of the pulse energy 
self-focuses. The present theory provides better agreement with results of numerical simulations than most of the theories used in the last decades for the self-focusing process of laser pulses. Comparison of the prediction of the present theory with Marburger's formula for the self-focusing distance shows an agreement within an error of $20 \%$ over a broad range of input powers of the pulse. Further comparisons with numerical simulations show that the pulse length as well as the on-axis intensity are predicted correctly within a $20 \%$ error too. We may emphasize that the comparisons are performed for collimated laser pulses, which is the worst case possible. Consideration of an external focusing would give even lower errors. Finally, we note that the present theory can be easily extended to consider losses and ionization of the gas medium.

\section{A Analysis of the diffraction term}

From Eqs. (11), (15) and (21) we can write

$$
\begin{aligned}
& u(\xi, r)=\frac{1}{a(\xi)} A(\xi) \exp \left(\rho^{2}\right) \exp \left(i b_{0} a(\xi) \rho^{2}\right) \\
& \times \quad \exp \left(i c(\xi) a(\xi)\left(2 \rho^{2} \exp \left(-2 \rho^{2}\right)-\exp \left(-2 \rho^{2}\right)\right)\right) \\
& \times \quad \exp \left(i \frac{\kappa \xi}{a^{3}(\xi)}\left(1-2 \rho^{2}\right)\right)
\end{aligned}
$$

where $A(\xi), a(\xi)$ and $c(\xi)$ are assumed to be variational parameters, and $\kappa$ is an unknown constant. By doing variational approximation similar to that one in section 2, but using the ansatz (45), one can show that in the critical regime $\left(P=P_{c r}\right)$

$$
\kappa=\text { constant } \times a^{\prime \prime}(0)=0 .
$$

Thus, in principle we can neglect corrections on the diffraction term and keep only the corrections in the nonlinear term in (45).

\section{References}

[1] J. Kasparian, M. Rodriguez, G. Méjean, J.. Yu, E. Salmon, H. Wille, R. Bourayou, S. Frey, Y.B A. Mysyrowicz, R. Sauerbrey, J.P. Wolf, and L. Wöste, Science 301, 61 (2003).

[2] R. R. Alfano, Supercontinuum Laser Source (Springer Verlag, New York, 1989). 
[3] J.H. Marburger, Prog. Quant. Electr. 4, 35 (1975).

[4] E. Dawes and J. Marburger, Phys. Rev. 179, 862 (1969).

[5] G. Fibich and A. Gaeta, Opt. Lett. 25, 335 (2000).

[6] G. Fibich, Opt. Lett. 21, 1735 (1996).

[7] G. Fibich and G. Papanicolaou, SIAM J. Appl. Math. 60, 183 (1999).

[8] R. W. Boyd, Nonlinear Optics (Academic Press, San Diego, USA, 2003).

[9] N. Aközbek, C. M. Bowden, A. Talebpour and S. L. Chin, Phys. Rev. E 61, 4540 (2000).

[10] J. Schwarz and J. C. Diels, Phys. Rev. A. 65, 013806 (2001).

[11] P. Sprangle, J. R. Peñano and B. Hafizi, Phys. Rev. E 66, 046418 (2002).

[12] S. Skupin, L. Bergé, U. Peschel, and F. Lederer, Phys.Rev.Lett. 93, 023901 (2004).

[13] M. Mlejnek, E. M. Wright, and J. V. Moloney, Opt. Lett., 23, 382 (1998).

[14] V.P. Kandidov, O.G. Kosareva, and A.A. Koltun, Quant. Electr. 33, 69 (2003).

[15] W. Liu, J. F. Gravel, F. Théberge, A. Becker and S. L. Chin, Appl. Phys. B (accepted for publication). 